\title{
Danas at persepsyon ng mga mag-aaral sa face-to-face at online learning: Isang integratibong rebyu
}

Dimasacat, Ainah Corina B.

Philippine Normal University, Manila, Philippines (dimasacat.acb@pnu.edu.ph)

Cacho, Reynald M.

Philippine Normal University South Luzon, Philippines (cacho.rm@pnu.edu.ph)

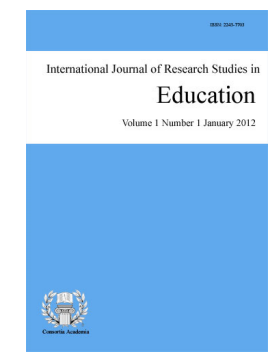

ISSN: $2243-7703$ Online ISSN: 2243-7711

\section{Abstract}

Ang kasalukuyang integratibong rebyu ng mga kaugnay na pag-aaral at literatura ay naglalayong alamin at unawain ang mga danas at persepsyon ng mga mag-aaral sa face-to-face at online learning. Mula sa nakalap na 20 pag-aaral gamit ang Google Scholar, 17 ang pinal na bilang ng mga sinuring papel batay sa mga pamantayang angkop sa proyektong na nais isagawa sa pagsasaliksik. Gamit ang mga pangunahing salik o dimensyong Course Structure/Organization, Social Interaction, Student Engagement, at Student Satisfaction, tinimbang at sinipat ang ugnayan ng mga nakalap na sanggunian. Ilan pang mga salik bukod sa mga naunang binanggit ang nakapagpaunlad sa pag-unawa sa tala ng mga kalahok sa bawat sinuring pag-aaral. Lumabas din sa kasalukuyang pagsusuri ang ilan pang mga posibleng gawing pokus ng mga susunod na pananaliksik gaya ng kung paano ba tinitingnan ng mga mag-aaral ang responsibilidad ng mga guro sa online learning. Gayundin ang pagsipat sa tunay na esensya ng palagiang isinusulong na kolaboratibong gawain sa kabila ng magkakaiba ang estilo sa pagkatuto ng mga mag-aaral. Inaasahang makatutulong ang rebyung ito upang magsagawa ng buong pananaliksik ang mga guro lalo na't patuloy ang pagbabagong-bihis at disenyo ng pagtuturo at pagkatuto sa Pilipinas.

Keywords: persepsyon, danas, online learning, face-to-face 


\section{Danas at persepsyon ng mga mag-aaral sa face-to-face at online learning: Isang integratibong rebyu}

\section{Panimula}

Kasabay ng mabilis na pagbabago ng mundo ang mga naiibang kahingiang kailangang punan ng lipunan. Patuloy na pinatutunayan ng teknolohiya ang mga benepisyong hatid nito sa buhay ng tao. Isa ang sektor ng edukasyon sa mga inaasahang makikinabang at tatanggap sa hamon ng inobasyon sa iba't ibang larangan. Gayunpaman, magkakaiba ang pagharap ng mga bansa sa mga makabagong pamamaraan sa edukasyon. Nangangailangan ito ng ganap na kahandaan at suporta mula sa mga miyembro ng naturang institusyon. At kaakibat ng mga pagbabago ang iba't ibang pagtingin, danas at persepsyon ng mga taong kailangang sumailalim dito, partikular na ang mga mag-aaral (Cacho, Avila, \& Villasenor, 2017).

Ayon sa pag-aaral nina Kulal at Nayak (2020), opinyon ng mga mag-aaral ang pinakamahalaga sa sistema ng edukasyon. Kung susundin ang ganitong pananaw, tutukoy ito sa alinmang pamamaraan --- tradisyunal o online learning. Tradisyunal o face-to-face ang pamamaraang nangangailangan ng presensya at pagkikita ng guro at mag-aaral sa isang tiyak na oras at lugar (University of the People, n.d.); habang online learning naman ang isang uri ng distance learning na ginagamit ng mga paaralang nakapaghahatid ng serbisyong edukasyonal gamit ang internet at mga platapormang online (Bates, 2016). Gayunpaman, sa kabila ng ganitong pagtingin, binanggit sa disertasyon ni Nwankwo (2015) na kulang na kulang ang mga pag-aaral na nabibigay-pokus sa danas at persepsyon ng mga mag-aaral sa online learning. Hindi gaanong nabibigyang-pansin ang boses ng mga mag-aaral, at patuloy lamang ang pagdami ng mga pananaliksik na may tuon sa iba pang aspeto ng online learning, ngunit hindi tungkol sa mag-aaral. Ang ganitong kakulangan sa batis ng kaalaman at pananaliksik tungkol sa nasabing paksa ang kailangang tugunan dahil kung pagbabatayan ang pahayag sa disertasyon ni La Piana (2014) na may tuon naman sa pag-aaral ng wika sa distance learning, makatutulong ang pagsasaliksik sa persepsyon ng mga mag-aaral upang mas maunawaan pa sila ng kanilang mga guro. Bunga nito, mas makaaagapay ang guro sa mag-aaral na harapin at pagtagumpayan ang mga pagsubok sa pagkatuto. Kaugnay nito ang tuon ng kasalukuyang rebyu. Sa pangangalap ng mga kaugnay na pag-aaral tungkol sa persepsyon at danas ng mga mag-aaral sa face-to-face at online learning, mas mauunawaan ng mga mananaliksik ang iba't ibang pagtingin ng mga kalahok mula sa iba't ibang konteksto at sitwasyon. Makatutulong ang pagtitimbang sa mga tala ng iba't ibang naunang mananaliksik nang sa gayo'y matugunan ang mga layunin ng sariling proyektong nais isagawa ng kasalukuyang pananaliksik. Kalaunan, sa tulong ng rebyung ito, mapaghahambing ng mga mananaliksik sa bubuuing pag-aaral ang danas at persepsyon ng mga mag-aaral sa face-to-face at online learning.

\section{Metodolohiya}

Ang kasalukuyang rebyu ng mga pag-aaral at literatura ay bahagi ng proseso ng pagbuo ng isang pananaliksik. Umiikot ito sa mga paksa tungkol sa persepsyon at danas ng mga mag-aaral sa face-to-face, online learning, o kumbinasyon ng dalawa. Pangunahing gamit ang Google Scholar upang makapangalap ng mga kaugnay na sanggunian. Upang maging mas tiyak sa paghahanap ng mga kaugnay na pag-aaral, ginamit ang mga katagang "Students' perceptions on online learning"; "students' perception on face-to-face and online learning"; "students' perceptions and experiences on online learning and face-to-face." Lubhang marami ang mga pag-aaral na lumabas kung kaya pinili ang mga titulong nalathala mula taong 2011 hanggang 2021. Mas nangunguna rin ang mga pag-aaral tungkol sa blended learning kung kaya kinailangang umabot hanggang pahina 10 pataas ng Google Scholar upang makapangalap ng sapat na bilang ng pag-aaral sa paksang tuon ng pagsusuri. Sa kabuuan, 20 pag-aaaral ang nakalap: lima (5) ang tungkol sa face-to-face at online learning; 11 ang tungkol sa online learning; isa (1) ang tungkol sa tradisyunal na paraan ngunit tuon sa paggamit ng mga platapormang online; isa (1) tungkol sa pag-aaral ng wikang Ingles sa konteksto ng Facebook; at isang (1) tungkol sa pagkakaiba ng pagtuturo ng 
wikang banyaga at iba pang asignatura sa isang birtwal na paaralan. Isang pag-aaral lamang ang ginawa sa Pilipinas, na nakatuon naman sa pagtuturo ng Filipino sa isang Open University. Napagpasyahan sa pagsasaliksik na ito na magpokus sa mga pag-aaral sa ilalim ng paksang face-to-face at online learning, online learning, at ang isang pag-aaral tungkol sa isang birtwal na paaralan. Mula 20 ay naging 17 na lamang ang mga pag-aaral na susuriin.

\section{Pagtalakay}

Sa paghahambing sa mga nakalap at sinuring pag-aaral, lumabas na pinakamarami ang nailathala sa mga taong 2011, 2013 at 2020 (tatlong pag-aaral sa bawat taon) at nangunguna ang bansang Amerika sa bilang ng mga nailathalang pag-aaral (walong pag-aaral), sinusundan ng bansang Australia (tatlong pag-aaral). Apat na bansang Asyano (India, Taiwan, Indonesia at UAE) ang kabilang sa listahan at ang may pinakamaraming nailathala ay bansang India (dalawang pag-aaral).

\section{Table 1}

Summary of studies about students' perceptions, and experiences in online learning, and face-to-face learning

\begin{tabular}{|c|c|c|c|c|}
\hline Author(s) \& Year & Country & Methodology & Participants & Focus of study \\
\hline Armstrong, 2011 & USA & Qualitative & University students & Online learning and instructional tools \\
\hline Bali and Liu, 2018 & Taiwan & Quantitative & University students & Face-to-face and online learning \\
\hline $\begin{array}{l}\text { Fidalgo, Thormann, } \\
\text { Kulyk, and Lencastre, } \\
2020\end{array}$ & UAE & Quantitative & University students & Online learning across three countries \\
\hline $\begin{array}{l}\text { Gray and DiLoreto, } \\
2016\end{array}$ & USA & Quantitative & Graduate students & $\begin{array}{l}\text { Students satisfaction and perceived } \\
\text { learning in online classes }\end{array}$ \\
\hline Huss and Shannon, 2013 & USA & Mixed Methods & University students & $\begin{array}{l}\text { Creation of action research from } \\
\text { students' feedback }\end{array}$ \\
\hline Kemp and Grieve, 2014 & Australia & Mixed methods & University students & opinions and test performance \\
\hline $\begin{array}{l}\text { Khan, Vivek, Nabi, } \\
\text { Kojah, and Tahir, } 2020\end{array}$ & India & Quantitative & University students & COVID-19 context \\
\hline Kulal and Nayak, 2020 & India & Quantitative & $\begin{array}{l}\text { Post-graduate \& graduate } \\
\text { students \& teachers }\end{array}$ & $\begin{array}{l}\text { Students' and teachers' perceptions in } \\
\text { online learning }\end{array}$ \\
\hline La Piana, 2014 & USA & Quantitative & $\begin{array}{l}\text { University \& community } \\
\text { college students }\end{array}$ & $\begin{array}{l}\text { Language learning in online class and } \\
\text { face-to-face setting }\end{array}$ \\
\hline $\begin{array}{l}\text { Lowenthal and Dunlap, } \\
2018\end{array}$ & USA & Mixed methods & Graduate students & $\begin{array}{l}\text { instructional strategies to establish social } \\
\text { presence }\end{array}$ \\
\hline $\begin{array}{l}\text { Mather and Sarkans, } \\
2018\end{array}$ & Canada & Qualitative & $\begin{array}{l}\text { Community College } \\
\text { students }\end{array}$ & $\begin{array}{l}\text { Comparison between online learning and } \\
\text { face-to-face learning }\end{array}$ \\
\hline Nwankwo, 2015 & USA & Qualitative & University students & Online learning \\
\hline $\begin{array}{l}\text { Oliver, Kellog, } \\
\text { and Patel, } 2012\end{array}$ & USA & Mixed Methods & $\begin{array}{l}\text { High school students and } \\
\text { teachers }\end{array}$ & $\begin{array}{l}\text { Differences between online FL } \\
\text { instruction and other subject areas in a } \\
\text { virtual school }\end{array}$ \\
\hline $\begin{array}{l}\text { Otter, Seipel, Graeff } \\
\text { Alexander, Boraiko, } \\
\text { Gray, Petersen, and } \\
\text { Sadler } 2013\end{array}$ & USA & Quantitative & $\begin{array}{l}\text { University students and } \\
\text { teachers }\end{array}$ & $\begin{array}{l}\text { Students' and faculty's perceptions in } \\
\text { face-to-face and online learning }\end{array}$ \\
\hline Rojabi, 2020 & Indonesia & Quantitative & University students & Use of microsoft teams \\
\hline $\begin{array}{l}\text { Tucker, Halloran, and } \\
\text { Price, } 2013\end{array}$ & Australia & Quantitative & University students & On teaching in online learning \\
\hline Yee, 2011 & Australia & Mixed methods & University students & $\begin{array}{l}\text { Malaysian students' perspectives in an } \\
\text { Australian university }\end{array}$ \\
\hline
\end{tabular}

Kung pagtutuunan ng pansin ang nilalaman ng mga kaugnay na pag-aaral, matutuklasan na marami ang tumatalakay sa persepsyon at danas ng mga mag-aaral sa online learning. Umiikot ang mga ito sa iba't ibang pokus at dimensyon: sa panahon ng COVID-19 (Khan, Vivek, Nabi, Khojah, \& Tahir, 2020); sa kagamitang panturo (Armstrong, 2011); estratehiyang panturo sa online classroom (Lowenthal \& Dunlap, 2018); gamit ng isang partikular na online platform (Rojabi, 2020); tungkol sa pagtuturo ng guro (Tucker, Halloran, \& Price, 2013); at pangkalahatang pagsusuri sa persepsyon ng mga mag-aaral (Nwankwo, 2015; Fidalgo, Thormann, Kulyk, \& Lencastre, 2020; Kulal \& Nayak, 2020; Yee, 2011; Huss \& Shannon, 2013). Lubha itong marami kung ikukumpara sa mga pag-aaral na tumatalakay naman sa persepsyon ng mga mag-aaral sa face-to-face at online learning (Mather \& Sarkans, 2018; La Piana, 2014; Bali \& Liu, 2018; Otter, Seipel, Graeff, Boraiko, Gray, Petersen, \& Sadler, 2013; Kemp \& Grieve, 2014). Kung kaya, upang mas maging malinaw ang rebyu sa mga pag-aaral, hinati sa mga sumusunod na salik o dimensyon ang pagtalakay: Course Structure/Organization (Gray \& 
Dimasacat, A. C. B., \& Cacho, R. M.

DiLoreto, 2016), Social Interaction (Gray \& DiLoreto, 2016; Bali \& Liu, 2018), Student Engagement (Gray \& DiLoreto, 2016), Student Satisfaction (Gray \& DiLoreto, 2016; Bali \& Liu, 2018), at Iba pang salik. Lalamanin ng seksyon ng "Iba pang salik" ang mga natuklasang tala na labas sa naunang apat na dimensyon. Narito ang mga nangibabaw na tema mula sa mga tala at resulta.

\subsection{Course Structure and Organization}

Nakasaad sa artikulong Measuring Student Perceptions in Web-Based Courses: A Standards-Based Approach ang pagpapakahulugan sa course structure/organization bilang "Policies and procedures that support and relate to the teaching/learning process, including course objectives, availability of library resources, types of materials provided to students, response time to students, and student expectations," (Jurczyk, Kushner-Benson, \& Savery, 2004, p. 2). Dagdag pa nina Gray at DiLoreto (2016), pumapasok din dito ang papel ng guro sa pagbibigay ng malinaw na detalye tungkol sa mga dapat asahan sa klase, petsa ng mga takdang-gawain, pagtatasa at rubrik, at mga sangguniang makatutulong sa pagkatuto ng mga mag-aaral. Isa ito sa mga itinuturing na mahahalagang salik na nakaaapekto sa persepsyon ng mga mag-aaral sa online learning (Moore, 1991 as cited in Gray \& DiLoreto, 2016). Mahihinuha mula sa mga binanggit na tala ang kahalagahang ibinibigay sa kaangkupan at kaayusan ng pagpaplano ng klase. Ito ang nagsisilbing patnubay sa mga mag-aaral kung ano ang mga dapat asahan sa magiging kabuuang danas ng pagkatuto. Samakatwid, mahihiwatigang kailangang lamanin ng estruktura at organisasyon ng kurso ang mga elementong posibleng makatulong at makapag-ambag sa magiging persepsyon at danas ng mag-aaral.

\subsection{Social Interaction}

Batay sa artikulo ni Okita (2012), mahalaga ang papel na ginagampanan ng social interaction sa pagkatuto. Dagdag pa niya, "interacting with other people has proven to be quite effective in assisting the learner to organize their thoughts, reflect on their understanding, and find gaps in their reasoning." Isa ito sa mga kaparaanang kailangang gawin sa klase upang mas maging makabuluhan ang proseso ng pagkakatuto, mapa-face-to-face o online learning man ito. Sa pag-aaral ni Ituma (2011) na pinamagatang "An evaluation of student'perceptions and engagement with e-learning components in a campus-based university," binanggit niya na nakapaghahatid ng bago at mas maayos na oportunidad ng interaksyon, engagement at pagkatuto ang e-learning para sa mga mag-aaral. Kaugnay nito, sinusuportahan ng ilang pag-aaral ang pagtalakay sa mga paraan upang mas mapagyaman ang interaksyon sa online learning, at maging ang mga positibong manipestasyon nito (Lowenthal \& Dunlap, 2018; Huss \& Eastep, 2013; Rojabi, 2020; Bali \& Liu, 2018).

Mayroon ding teoryang nakapokus sa interaksyong esensyal sa distance learning, ang Interaction Theory $\mathrm{ni}$ Moore (1989) na nagpapahayag ng kahalagahan ng interaksyong mag-aaral - nilalaman, interaksyong mag-aaral-guro, at interaksyong mag-aaral - mag-aaral. At kapag pinag-uusapan ang konsepto ng interaksyon, hindi maaaring hindi mabanggit ang konsepto ng komunikasyon. Batay pa nga kina Richardson, Swan, Lowenthal at Ice (2016) na binanggit sa pag-aaral nina Lowenthal at Dunlap (2018), nakadepende ang edukasyon sa mabisang komunikasyon. Ngunit nagbabago ang komunikasyon kapag hindi na direkta o may namamagitan na sa pagpapalitan ng mensahe. Dahil sa pag-aaral ni Armstrong (2011), naiwan sa kasalukuyang pananaliksik ang tanong na, "nakaaapekto ba sa inaasahang kahalagahang akademiko ang dalas ng interaksyon sa pagitan ng guro at mag-aaral? Kung oo, gaano kadalas upang maituring na magbubunsod ang gayong danas sa maayos at sapat na kahalagahang akademiko? May hangganan kayang dapat isaisip at maaaring subuking abutin?” Ito ang mga katanungang maaari pang tuklasin dahil ilan ding mga pag-aaral ang nagpahayag ng nagtutunggaliang persepsyon mula sa mga mag-aaral pagdating sa usapin ng interaksyon at komunikasyon. Dalas o kaledad? Ano ang pamantayan upang masabing produktibo at mabisa ang interaksyon?

\subsection{Student Engagement}

Madalas na naiuugnay ang salik ng student engagement sa social interaction batay sa mga naunang kaugnay

92 Consortia Academia Publishing (A partner of Network of Professional Researchers and Educators) 
Danas at persepsyon ng mga mag-aaral sa face-to-face at online learning: Isang integratibong rebyu

na pag-aaral (Armstrong, 2011; Oliver, Kellog, \& Patel, 2012; Kemp \& Grieve, 2014). Ngunit sa pag-aaral nina Gray at DiLoreto (2016), binanggit nila ang pagpapakahulugan nina Bomia, Beluzo, Demeester, Elander, Johnson, \& Sheldon (1997, p. 294) sa student engagement bilang "students' willingness, need, desire, and compulsion to participate in, and be successful in, the learning process." Habang sa artikulo naman nina Mandernach, Donnelli-Sallee, \& Dailey-Hebert (2011), inilahad nila ang mga apektib na salik na may kaugnayan sa student engagement tulad ng personalidad, motibasyon, effort, at kumpiyansa sa sarili. Sa isang kaugnay na pag-aaral (Kemp \& Grieve, 2014) tungkol sa opinyon at test performance ng mga mag-aaral sa tradisyunal at online learning, lumabas sa resulta na mas pinipili pa rin ang face-to-face kumpara sa online learning. Paliwanag ng mananaliksik, mas nararamdaman ng mag-aaral ang pagiging engaged sa mga talakayan sa tradisyunal na klase kaysa sa online discussion board. Salungat ito sa persepsyon ng mga kalahok na mag-aaral sa pag-aaral nina Mather at Sarkans (2018), na nagsabing, nakatutulong din ang mga kagamitang pampagkatuto at mga discussion forums upang mas maging engaged sila sa klase sa online. At sa pakikilahok ng mga mag-aaral sa mga opsyonal na gawain sa online learning mahihiwatigan ang kanyang motibasyon sa pag-aaral (Oliver, et al., 2012).

\subsection{Student Satisfaction}

Sa disertasyon ni Nwankwo (2015), binanggit ang pahayag nina Kuo, Walker, Belland, at Schroder (2013) na nagbibigay-tuon sa usapin kung bakit dapat pagtuunan din ng pansin ang satispaksyon ng mga mag-aaral. Anila, among the attitudinal constructs, student satisfaction, referring to student perceptions of learning experiences and perceived value of a course, may be particularly worthy of investigation (p. 17). Naglatag naman si Lo (2010) ng mga maaaring sukatan ng pag-alam sa satispaksyon ng mga mag-aaral na binanggit sa pag-aaral nina Bali \& Liu (2018): satisfaction with instructors' directions and support, satisfaction with own commitment to learning, satisfaction with course policies (p. 3). Tinalakay pa rin sa ilang mga pananaliksik ang iba't ibang usapin tungkol sa satispaksyon ng mga mag-aaral, mapa-face-to-face at online learning man ito (Paechter, Maier, \& Macher, 2010; La Piana, 2014; Gunawaderna \& Zittle as cited in Bali \& Liu, 2018; Otter, Seipel, Graeff, Alexander, Boraiko, Gray, Petersen, \& Sadler, 2013; Ladyshewsky, 2013 as cited in Tucker, Halloran, \& Price, 2013).

Mula sa mga tala at resulta ng mga naunang mga mananaliksik, nakumpirma ng kasalukuyang pagsasaliksik na kung mayroon mang salik na laging nababanggit tungkol sa persepsyon ng mga mag-aaral, ito ay ang satispaksyon. Napagtanto rin na ang unang tatlong dimensyong binanggit, ang Course Structure, Social Interaction, at Student Engagement, ay mga salik na nakapag-aambag sa satispaksyon ng mag-aaral. Samakatwid, mahalagang malaman at masuri ang satispaksyon dahil batay na rin sa mga naunang pag-aaral, lantad ang kaugnayan nito sa kabuuang danas at persepsyon ng mga mag-aaral.

\subsection{Iba pang salik}

Sa ilang mga pag-aaral, malinaw ring inilatag ang iba pang mga salik na nangingibabaw sa online at face-to-face learning batay sa persepsyon ng mga kalahok (Fidalgo, Thormann, Kulyk, \& Lencastre, 2020; Kulal \& Nayak, 2020; Mather \& Sarkans, 2018; Bali, \& Liu, 2018; Rojabi, 2020). Isa na rito ang usapin ng paggamit ng mga kagamitan lalo na sa online learning na ayon sa pag-aaral ni Armstrong (2011), mas binibigyang-halaga ng mga mag-aaral ang kaparaanan sa pagpapagamit ng mga online learning tools, kaysa sa mismong mga kagamitan at instrumento. Samakatwid, nasa implementasyon ang kahalagahan, hindi sa kagamitang panturo o pampagkatuto. Nabanggit din ang konsepto ng social presence na batay sa masusing pagsusuri nina Lowenthal at Dunlap (2018) ay isang popular na construct na ginagamit upang ilarawan kung paano nakikipag-ugnayan ang mga tao o mag-aaral sa mga klaseng online. Hindi rin maaaring mawala ang mga pagpapahayag tungkol sa mga positibo at negatibong aspeto ng face-to-face at online learning. May pag-aaral na nagsasabing mas madali ang interaksyon sa online learning kaysa face-to-face (Rojabi, 2020), habang sumasalungat dito ang ilan dahil kulang o di kaya nama'y huli na kung makipag-ugnayan at magbigay ng feedback at ebalwasyon ang mga guro sa online learning (Mather \& Sarkans, 2018). Nangunguna namang bentahe ng online learning kaysa face-to-face ang kaginhawaang hatid nito sa mga mag-aaral dahil hindi na sila malilimitahan ng ilang mga balakid sa pagkatuto gaya ng heyograpikal na lokasyon at oras kung kailan nila ito maaaring gawin (Khan, Vivek, Nabi, Khojah, \& Tahir, 2020; 
Mather \& Sarkans, 2019). Gayunpaman, ang ilang mga positibong hatid ng online learning ay maituturing ding pagsubok para sa iba. Sa mapanuring disertasyon ni La Piana (2014), kumprehensibo inilatag ang kahalagahang alamin ang sariling estilo sa pagkatuto ng mga mag-aaral bilang batayan ng kahandaan sa pag-aaral ng ikalawa o banyagang wika sa online learning.

Binigyang- diin din ang aniya'y reklamo nina Yadin at Or-Bach (2010) tungkol sa patuloy na pagbibigay-pokus ng mga pag-aaral sa usapin ng kolaborasyon habang hindi gaanong nabibigyang-pansin ang independent study. Malaking papel ang ginagampanan dito ng multiple intelligences (Gardner, 1983) partikular na ang intrapersonal at interpersonal intelligences na ginamit din ni La Piana sa pag-aaral. Angkop ba na laging mga kolaboratibong gawain ang ibigay sa mga mag-aaral upang magkaroon sila ng interaksyon sa mga kaklase nila? O maaari ring tingnan ang esensya ng pagpapagawa ng mga aktibidad na mag-aaral lamang ang makatutulong sa kanyang sarili? Maiuugnay dito ang inilatag na pagsubok na kinaharap ng mga mag-aaral sa mga pag-aaral nina Mather at Sarkans (2018) at Huss at Eastep (2013) na kapwa face-to-face at online learning, mapanghamon ang mga kolaboratibong gawain dahil sa iba't ibang kadahilanan. Magkakaiba rin ang persepsyon ng mga mag-aaral sa papel na ginagampanan ng mga guro sa online learning. Ngunit ang pinakainteresanteng resulta ng kaugnay na pag-aaral tungkol sa salik na ito ay ang pagtingin ng mga mag-aaral na hindi gaanong mabigat ang responsibilidad ng mga guro sa online learning (Otter, Seipel, Graeff, Alexander, Boraiko, Gray, Petersen, \& Sadler, 2013). Dagdag pa, kung mas may nahihirapan, ito ay ang mga mag-aaral dahil anila, ang bigat ay nasa kanilang balikat na at hindi masyadong kailangang magbigay ng effort at oras ang mga guro sa online learning.

Ilan lamang ang mga ito sa magkakaibang pananaw, pagtingin, at persepsyon ng mga mag-aaral tungkol sa iba't ibang salik na naglalarawan sa face-to-face at online learning. Mahalagang tingnan, sipatin at talakayin ang mga ito dahil ayon nga kina Huss at Eastep (2013):

Closing the gap between those perceptions and the realities they actually encounter within various online classes will be instrumental in helping us (and perhaps many of our colleagues) develop courses that provide the flexibility students desire while maintaining a necessary sense of "connectedness" with our institution and our faculty (p. 15).

\section{Konklusyon}

Kaakibat ng pagtanggap sa hamon ng inobasyon at paglinang sa kasanayang akademiko gamit ang mga pamamaraang teknolohikal, ang pagsasaalang-alang sa danas at persepsyon $\mathrm{ng}$ mga miyembro ng sistema $\mathrm{ng}$ edukasyon. Isa lamang ang pagtukoy sa pananaw at pagtingin ng mga mag-aaral tungkol sa online learning, at ang kalauna'y paghahambing nito sa danas nila sa face-to-face, sa mga hakbang na maaaring isagawa upang mas maunawaan kung ano ang kanilang pinagdadaanan at pangangailangan. Sa pag-alam ng pangangailangan ng mga mag-aaral sa naiibang disenyo ng pagtuturo at pagkatuto, magagabayan nito ang mga guro at administrador ng mga paaralan sa mga kakailanganing pagsasaayos at pagbabago. Matutulungan nito ang mga guro na unawain at kalauna'y timbangin ang iba't ibang pagtingin at danas ng mga mag-aaral sa kanilang klase batay sa mga inilatag na salik ng kasalukuyang papel. Ang resulta ng rebyung ito ay maaari ring maging salalayan sa paggawa ng sariling papel pananaliksik ng mga guro tungkol sa kaugnay na paksa. Sa pamamagitan ng kontekstwalisasyon, masusuportahan o mapabubulaanan ng kani-kanilang pagsasaliksik ang mga naunang tala na binanggit sa kasalukuyang papel. Sa pagbabasa ng rebyung ito ay mababatid din ng mga mag-aaral ang maaari'y kapareho o naiibang danas ng mga kapwa nila mag-aaral mula sa iba't ibang bansa.

Lumabas sa kasalukuyang rebyu at pagsusuri na napakaraming salik ang maaaring tingnan at sipatin upang mas mahimay ang danas at persepsyon ng mga mag-aaral sa face-to-face at online learning. May mga interesanteng puntos din na maaari pang pagtuunan ng pansin upang makita ang ibang anggulo ng pag-unawa sa mga mag-aaral gaya ng kung paano nga ba nila tinitingnan ang trabaho at responsibilidad ng guro sa online learning (Otter, Seipel, Graeff, Alexander, Boraiko, Gray, Petersen, \& Sadler, 2013), at ang saysay ng pagsusulong at pagsasagawa ng mga kolaboratibong gawain, sa kabila ng magkakaibang kakayahan, kasanayan 
Danas at persepsyon ng mga mag-aaral sa face-to-face at online learning: Isang integratibong rebyu

at estilo ng mag-aaral sa pagkatuto (Yadin \& Or-Bach, 2010 in La Piana, 2014). Sa 17 sinuring pag-aaral, isa lamang ang kumuha ng mga kalahok mula sa antas hayskul kung kaya mainam na magsagawa pa ng ibayong pag-aaral na mangangalap ng datos mula sa mga mag-aaral na kabilang sa antas na ito. Sa tulong ng ganitong uri ng pag-aaral, malalaman ang antas ng kahandaan ng mga mag-aaral sa mga pagbabagong hatid ng paggamit ng teknolohiya sa edukasyon. Ito ay dahil lalo nasa kasalukuyang sitwasyon, mas lumaganap ang kahingian sa paglipat sa online learning dahil sa pandemya ng COVID-19. Kapaki-pakinabang na isakatuparan ang ganitong klase at tuon ng pag-aaral lalo na at humaharap at patuloy na sasailalim sa malaking pagbabago at hamon ang sistema ng edukasyon sa Pilipinas dahil sa epekto ng iba't ibang pangyayaring panlipunan. Ngayon, higit kailanman, kailangang bigyang-daan at pagyamanin pa ang mga pag-aaral na nagbibigay-halaga sa boses at pulso ng mga mag-aaral. Dahil ang mga mag-aaral ay hindi lamang makikinabang; bagkus ay dapat katuwang ng guro at paaralan sa pagtamo ng edukasyong de-kaledad, inobatibo at makatao, para sa lipunan.

\section{Talasanggunian}

Armstrong, D. (2011). Students' perceptions of online learning and instructional tools: A qualitative study of undergraduate students use of online tools. TOJET: The Turkish Online Journal of Educational Technology, 10(3). https://files.eric.ed.gov/fulltext/EJ944973.pdf

Bali, S., \& Liu, M. C. (2018). Students perceptions toward online learning and face-to-face learning courses. Journal of Physics: Conference Series. https://doi.org/10.1088/1742-6596/1108/1/012094

Bates, T. (2020, May 10). Online learning for beginners: 1. What is online learning? Tony Bates. https://www.tonybates.ca/2016/07/15/online-learning-for-beginners-1-what-is-online-learning/

Cacho, R. M., Avila, R. V., \& Villaseñor, E. S. (2017). What's the tab's apps? Piloting low-priced-tablet-aided course delivery in teacher education. International Journal of Emerging Technologies in Learning, 12(10). https://doi.org/10.3991/ijet.v12.i10.7162

Fidalgo, P., Thormann, J., Kulyk, O., \& Lencastre, J. A. (2020). Students' perceptions on distance education: A multinational study. International Journal of Educational Technology in Higher Education, 17(1). https://doi.org/10.1186/s41239-020-00194-2

Gray, J., \& DiLoreto, M. (2016). The effects of student engagement, student satisfaction, and perceived learning in online learning environments. NCPEA International Journal of Educational Leadership Preparations, 11(1). https://files.eric.ed.gov./fulltext/EJ1103654.pdf

Huss, J. A., \& Eastep, S. (2013). The perceptions of students toward online learning at a Midwestern University: What are students telling us and what are we doing about it? Inquiry in Education, 4(2). https://files.eric.ed.gov/fulltext/EJ1171787.pdf

Ituma, A. (2011). An evaluation of students' perceptions and engagement with e-learning components in a campus based university. Active Learning in Higher Education, 12(1), 57-68. https://doi.org/10.1177/1469787410387722

Jurczyk, J., Kushner Benson, S. N., \& Savery, J. R. (2004). Measuring student perceptions in web-based courses: A standards-based approach. Online Journal of Distance Learning Administration, 7(4). https://www.westga.edu/ distance/ojdla/winter74/jurczyk74.htm

Kemp, N., \& Grieve, R. (2014). Face-to-face or face-to-screen? Undergraduates' opinions and test performance in classroom vs. online learning. Frontiers in Psychology, 5. https://doi.org/10.3389/fpsyg.2014.01278

Khan, M. A., Vivek, V., Nabi, M. K., Khojah, M., \& Tahir, M. (2020). Students' perception towards e-learning during COVID-19 pandemic in India: An empirical study. Sustainability, 13(1), 57. https://doi.org/10.3390/su13010057

Kulal, A., \& Nayak, A. (2020). A study on perception of teachers and students toward online classes in Dakshina Kannada and Udupi District. Asian Association of Open Universities Journal, 15(3), 285-296. https://doi.org/10.1108/aaouj-07-2020-0047

La Piana, N. (2014). Comparing students' perceptions of online language learning to traditional learning (Doctoral dissertation). https://digitalcommons.liberty.edu/cgi/viewcontent.cgi?article=1931\&context=doctoral 
Dimasacat, A. C. B., \& Cacho, R. M.

Lewison, K. (2020, October 15). Facts: Is online learning as good as face-to-face learning? University of the People. https://www.uopeople.edu/blog/online-learning-good-+as-face-to-face-learning/

Lo, C. (2010). How student satisfaction factors affect perceived learning. Journal of the Scholarship of Teaching and Learning, 10(1), 47-54. https://files.eric.ed.gov/fulltext/EJ882125.pdf

Lowenthal, P. R., \& Dunlap, J. C. (2018). Investigating students' perceptions of instructional strategies to establish social presence. Distance Education, 39(3), 281-298. https://doi.org/10.1080/01587919.2018.1476844

Mandernach, B. J., Donnelli-Sallee, E., \& Dailey-Hebert, A. (2018). Assessing course student engagement. Academia. https://www.academia.edu/1807421/Assessing_course_student_engagement

Mather, M., \& Sarkans, A. (2018). Student perceptions of online and face-to-face learning. International Journal of Curriculum and Instruction, 10(2), 61-76. https://files.eric.ed.gov/fulltext/EJ1207234.pdf

Nwankwo, A. (2015). Students' learning experiences and perceptions of online course content and interactions (Doctoral dissertation). https://scholarworks.waldenu.edu/cgi/viewcontent.cgi?article=1187\&context=dissertations

Okita, S. (2012). Social interactions and learning. Retrieved from In N. M. Seel (Eds.), Encyclopedia of the Sciences of Learning. Springer. https://doi.org/10.1007/978-1-4419-1428-6_1770

Oliver, K., Kellogg, S., \& Patel, R. (2012). An investigation into reported differences between online foreign language instruction and other subject areas in a virtual school. CALICO Journal, 29(2), 269-296. https://doi.org/10.11139/cj.29.2.269-296

Otter, R. R., Seipel, S., Graeff, T., Alexander, B., Boraiko, C., Gray, J., Petersen, K., \& Sadler, K. (2013). Comparing student and faculty perceptions of online and traditional courses. The Internet and Higher Education, 19, 27-35. https://doi.org/10.1016/j.iheduc.2013.08.001

Petras, J. (2012). E-Filipino: Ang pagtuturo at pagkatuto ng/sa wikang Filipino sa sistemang open and distance learning [E-Filipino: Teaching and learning in/of the Filipino language in an open system and distance learning]. DALUMAT E-Journal, 3(1-2). http://ejournals.ph/form/cite.php?id=6215

Rojabi, A. R. (2020). Exploring EFL students' perception of online learning via microsoft teams: University level in Indonesia. English Language Teaching Educational Journal, 3(2), 163-173. https://doi.org/10.12928/eltej.v3i2.2349

Tucker, B., Halloran, P., \& Price, C. (2013). Student perceptions of the teaching in online learning: An Australian university case study. Research and Development in Higher Education: The Place of Learning and Teaching, 36th HERDSA Annual International Conference (pp. 470-484). Auckland, New Zealand: HERDSA.

Understanding Social Interaction. (n.d.). Retrieved April 2 2021, from Lumen Boundless Sociology. https://courses.lumenlearning.com/boundless-sociology/chapter/understanding-social-interaction/

Zamari, Z. M., Adnan, A. H. M., Idris, S. L., \& Yusof, J. (2012). Students' Perception of using Online Language Learning Materials. Procedia - Social and Behavioral Sciences, 67, 611-620. https://doi.org/10.1016/j.sbspro.2012.11.367 